\title{
Nonlinear Integrated Microwave Photonics
}

\author{
David Marpaung* and Benjamin J. Eggleton \\ Centre for Ultrahigh bandwidth Devices for Optical Systems (CUDOS), the Institute of Photonics and Optical Sciences (IPOS), \\ School of Physics, University of Sydney, NSW 2006, Australia \\ *e-mail: d.marpaung@physics.usyd.edu.au
}

\begin{abstract}
Harnessing nonlinear optical effects in a photonic chip scale has been proven useful for a number of key applications in optical communications. Microwave photonics can also benefit from the adoption of such a technology, creating a new concept of nonlinear integrated microwave photonics. Here, we discuss the potential of on-chip nonlinear processing towards the creation of robust and multifunctional microwave photonic (MWP) processors. We also highlight key recent results in the field, including frequency agile MWP filters and ultrawideband signal generators.
\end{abstract}

Keywords - analog signal processing; microwave photonics; photonic integrated circuits; $R F$ filters; $R F$ photonic filters

\section{INTRODUCTION}

Recently, there is a significant interest in exploiting photonic integrated circuits (PICs) for the processing of microwave signals [1]. The term integrated microwave photonics (IMWP) is used to describe research activities in this area. IMWP clearly promises a number of advantages compared to conventional fiber-based approach. The potential of integrating a number of different signal processing functionalities in a single chip will lead to significant reduction in size, weight, power consumption, and cost. Moreover, the ability to integrate various functions like modulation, passive signal processing, and detection in a single chip will lead to reduction in insertion losses, which is the key for MWP signal processor to compete with RF signal processor in terms of performance.

Thus far, IMWP has largely been limited to linear optical processing. It is interesting, however, to examine the potential of nonlinear optics in integrated platform for microwave signal processing. Optical nonlinearities (e.g., $\chi^{(2)}$ and $\left.\chi^{(3)}\right)$ [2], such as sum and difference-frequency generation (SFG/DFG), crossphase modulation (XPM), degenerate/non-degenerate fourwave mixing (FWM), and stimulated Brillouin scattering (SBS), are suitable candidates for MWP signal processing. In the context of optical signal processing, these nonlinear optics effects have efficiently been harnessed, both in fibers and in integrated devices, for a number of functionalities such as alloptical delay [3], all-optical switching and multiplexing [4], and wavelength multicasting [5].

In this paper, we look at the potential of combining linear and nonlinear optical effects in a chip scale to enable a number of functionalities such as agile microwave filtering and spectrum channelization, with an ultimate goal to achieve a general purpose reconfigurable analog signal processor. We then review a number of recent IMWP demonstrations that exploited on-chip SBS, FWM, and XPM, in various platforms such as chalcogenide glasses and silicon. Finally, we discuss the prospects and as well as the challenges that should be tackled in order for nonlinear IMWP to thrive.

\section{POTENTIAL}

The exponential growth in bandwidth demand of radio communications has put unprecedented challenges in the RF signal processing chain. Traditionally, strong emphasis in such a chain is put on digital signal processing (DSP). However, for increasing operating frequencies and larger bandwidths, a reliable analog signal processor (ASP) that can match the flexibility of a DSP is desired [6, 7]. Due to its high bandwidth and potential reconfigurability, IMWP processor is an attractive candidate for such a flexible ASP. However, current state-of the-art IMWP processors were developed only for very specific tasks, for example beamforming and filtering [8], frequency discrimination [9], or pulse shaping [10]. Thus, there is a lack of generality and multi-functionality of these processors. To emulate DSP, the IMWP processor has to be application agnostic, multi-purpose and programmable.

We believe that incorporating optical nonlinearities in an IMWP processor will open up the path towards a general purpose ASP. Gasulla and Capmany [11] have identified that a large number of MWP signal processing can be generalized as processing of a number copies of RF modulated optical signals using reconfigurable optical filters. Nonlinear optical processes that create new optical wavelengths such as FWM, SFG, or DFG are attractive candidates for generating such signal copies. In fact, this concept of wavelength multicasting has recently been implemented for MWP signal processing

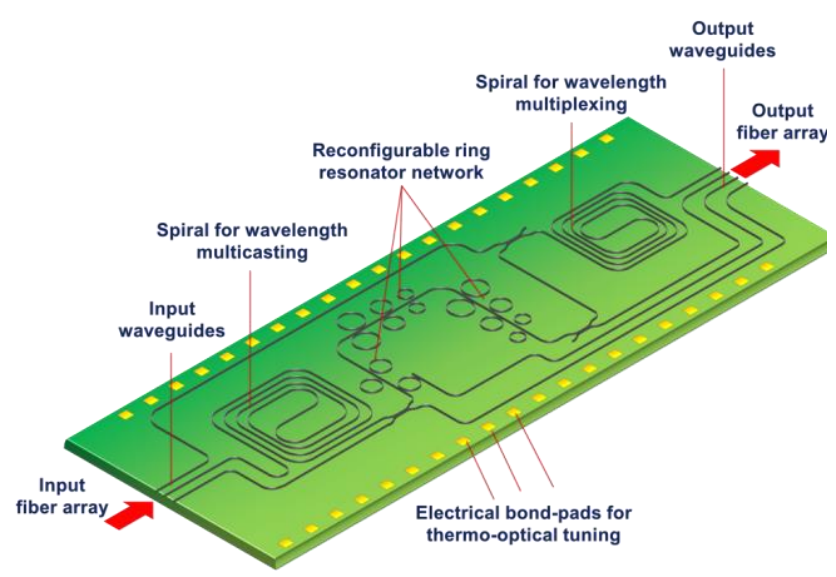

Fig. 1. (a) Illustration of the envisioned general purpose analog signal processor architecture. 
techniques such as channelization $[12,13]$ and reconfigurable filtering $[14,15]$. In [12-14] FWM processes in highly nonlinear fibers (HNLF) were used, while SFG and DFG in PPLN have been exploited in [15]. In the view of the general processor, these wavelength copies will subsequently be processed by a reconfigurable optical filter. The typical signal processing tasks carried out by these filters include tunable time delay, carrier phase tuning, and complex (amplitude and phase) filtering. These processed optical signals will then be recombined or directed to separate dedicated outputs for a photodetection process.

The key challenge here is to create a monolithic photonic chip that host the nonlinear optics for wavelength multicasting and the reconfigurable linear optical filtering. Fig. 1 depicts an illustration of a possible design of such a photonic chip in $\mathrm{Si}_{3} \mathrm{~N}_{4}$ technology. The chip consists of three main sections: two long spiral waveguides for wavelength multicasting and multiplexing based on cascaded FWM processes, and reconfigurable optical filter based on a network of ring resonators that can be tuned using thermo-optics effect. This ring network might host a mixture of all pass ring filters, adddrop rings, as well as Vernier (cascade of non-identical) rings which have been recently exploited for tunable delay lines in a multi-wavelength beamformer [16]. The programmability of the ring network will allow the chip to be reconfigured in real time, to synthesize different responses according to the user demand.

Obviously, other material platforms such silicon can also serve as good candidates to construct this ASP. However, $\mathrm{Si}_{3} \mathrm{~N}_{4}$ waveguides might be more suited for this purpose due to the following characteristics: ultra-low propagation losses (approx. $0.1 \mathrm{~dB} / \mathrm{cm}$ [17]) which is very important for MWP systems, moderately high optical nonlinearity (10 times of silica) [1821] and free from nonlinear losses such as two-photon absorption (TPA) and free-carrier effect (FCA) [19]. On top of this, $\mathrm{Si}_{3} \mathrm{~N}_{4}$ waveguides have shown compatibility with efficient thermo-optics tuning and is a CMOS compatible material. While silicon also tick some of the boxes and successful wavelength multicasting has been demonstrated in this platform [22, 23], the propagation loss of a typical SOI nanowires is relatively high $(2-3 \mathrm{~dB} / \mathrm{cm})$ [24] and they suffer from TPA and FCA $[4,19]$. These losses might be prohibitive for creating an efficient nonlinear IMWP processor.

\section{CURRENT StATUS}

Very recently, a number of nonlinear IMWP demonstrations have been shown with impressive performance. For example, on chip SBS has been harnessed for creating an ultra-high performance tunable notch filter, while FWM in silicon nanowire has been used in the context of a reconfigurable multi-tap MWP filter. We believe this development is a significant first step towards the realization of a general purpose processor. Here, we review some these demonstrations.

\section{A. On-chip SBS filters}

Stimulated Brillouin scattering (SBS) is a nonlinear scattering process where a pump wave, when encounter acoustic vibrations in the medium, gets backscattered, resulting

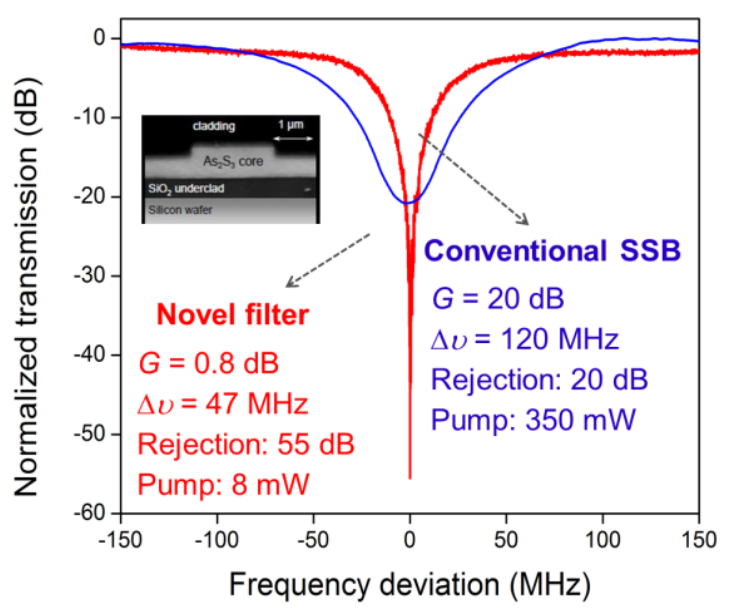

Fig. 2. Experimental result of MWP notch filter response using on-chip SBS with sub-1 dB gain. Blue trace indicates conventional filter using single sideband modulation and SBS loss spectrum [31]. Red trace indicates notch response obtained from novel complex sideband processing [34] and SBS gain spectrum. The novel technique leads to an increase in filter resolution (47 $\mathrm{MHz}$ full-width at half maximum) and ultra-high enhancement of the peak rejection $(>55 \mathrm{~dB})$, achieved with only $8 \mathrm{~mW}$ of pump power.

in Stokes and anti-Stokes waves. A probe, centered at the Stokes frequency, experiences gain when counter-propagated to the pump whereas a probe that is up-shifted with respect to the pump (anti-Stokes), experiences absorption. SBS has long been exploited in optical fibers for MWP signal processing $[25,26]$, but the recent demonstration of on-chip SBS [27] has finally enabled the control of this process in a compact photonic chip scale. The photonic chip was a $6.5-\mathrm{cm}$ long chalcogenide glass $(\mathrm{ChG}) \mathrm{As}_{2} \mathrm{~S}_{3}$ optical waveguide with a cross-section of $4 \mu \mathrm{m} \times 850 \mathrm{~nm}$ and a large SBS gain coefficient $\left(g_{\mathrm{B}} \sim 0.74 \times 10^{-9} \mathrm{~m} / \mathrm{W}\right)$. Using this photonic chip, key SBS RF signal processing such as tunable delay line [28] tunable bandpass [29] and notch [30,31] filtering have recently been demonstrated.

Recently, a remarkable progress has been made regarding MWP tunable notch filters based on SBS. A long standing problem in RF filters is to achieve wide frequency tuning simultaneously with high resolution filtering. For example, state-of-the-art absorptive band-stop filters are capable of a high peak attenuation $(>50 \mathrm{~dB})$ and high resolution $(<10 \mathrm{MHz}$ 3-dB isolation bandwidth measured from the passband) but have limited notch frequency tuning range, in the order of $1.4 \mathrm{GHz}$ [32]. MWP notch filters, on the other hand, are capable of multi-gigahertz tuning, but are often limited in peak attenuation $(<40 \mathrm{~dB})$ and resolution (gigahertz bandwidth instead of tens of megahertz). In [33], Marpaung et al. proposed a novel class of MWP notch filters with an ultrahigh peak rejection. In this novel scheme, an RF signal was encoded in the optical sidebands with unequal amplitudes and a phase difference using an electro-optic modulator (EOM). An optical filter (OF) was then used to equalize these sidebands amplitudes and to produce an anti-phase relation between them only in selected frequency region within the OF response. Upon photodetection, the beat signals generated from the mixing of the optical carrier and the two sidebands perfectly 


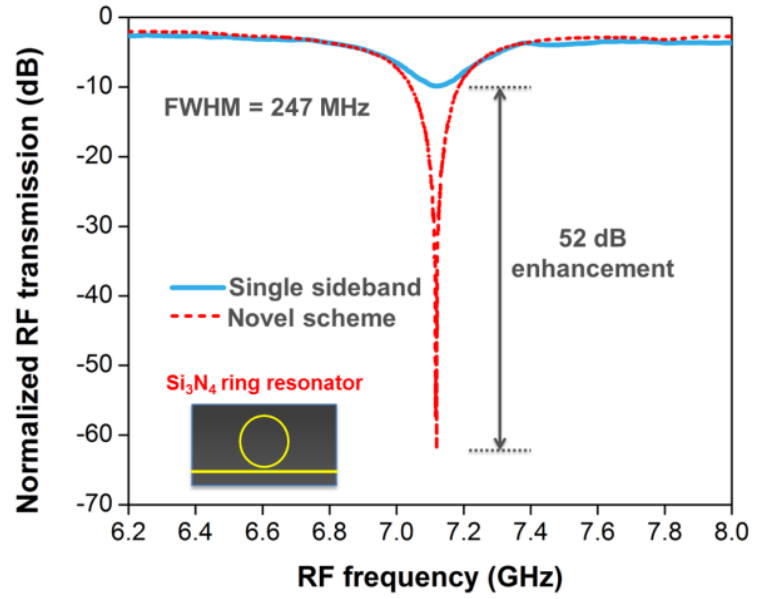

Fig. 3. Experimental result of MWP notch filter response using a low loss $\mathrm{Si}_{3} \mathrm{~N}_{4}$ ring resonator [35]. Implementing the complex sideband processing [33-35] led to a peak rejection enhancement of $>50 \mathrm{~dB}$ relative to conventional single sideband scheme.

cancel at a specific microwave frequency, forming a notch with an anomalously high stopband rejection. Using SBS gain spectrum in $650 \mathrm{~m}$ of optical fibers as the OF, they realized an MWP notch filter with record performance (a high selectivity of $\sim 10 \mathrm{MHz}$, an ultrahigh stopband rejection of $>60 \mathrm{~dB}$, wide continuous frequency tuning of $1-30 \mathrm{GHz}$, and flexible bandwidth reconfigurability $10-65 \mathrm{MHz}$ ). Very recently, this concept has also been implemented with SBS in compact $\mathrm{ChG}$ photonic chip. In [34], Marpaung et al. achieved a filter with an ultra-high suppression of more than $55 \mathrm{~dB}$, a high resolution of $47 \mathrm{MHz}$, and ultra-wide tunable central frequency of 1-30 GHz. Remarkably, this was achieved using an ultra-low SBS gain of $0.8 \mathrm{~dB}$, and a very low pump power of $7 \mathrm{~mW}$. This represents 50 times reduction in required pump power, relative to a conventional SBS notch filter generated using single-sideband modulation scheme [31]. The measured responses of the novel and conventional filters are shown in Fig. 2. We believe that these results will ease the implementation of low power nanophotonic devices as high performance RF notch filters and will change the landscape of the field of integrated microwave photonics.

The key advantage of the novel notch filter scheme is that it is applicable for a wide range of optical filters. Marpaung et al. also implemented this technique using a reconfigurable silicon nitride ring resonator [35]. They achieved peak rejection enhancement of more than $50 \mathrm{~dB}$ (Fig. 3). Such a high suppression would normally occur only when the resonator is critically coupled. But the novel technique allows one to achieve this high contrast independent of the ring coupling, thereby significantly relaxing the fabrication tolerance of such ring resonators.

\section{B. On chip XPM and FWM}

On-chip ultrafast Kerr nonlinearity has been exploited in the context MWP signal processing for ultra-wideband (UWB) signal generation [36] and reconfigurable multi-tap filtering [37]. In [36], Tan et al. combine the effects of cross-phase modulation (XPM) and birefringence in an $\mathrm{As}_{2} \mathrm{~S}_{3}$ rib waveguide to generate polarity-inverted UWB monocycles with a single optical carrier. The high Kerr-nonlinearity of $\mathrm{ChG}$ in a chip platform enables efficient XPM in a short length of $7 \mathrm{~cm}$. The combination of XPM and birefringence essentially acts as an all-optical differentiator that converts a train of Gaussian pulses into a train of monocycle electrical pulses after photo-detection process. Using this technique, Tan et al. demonstrated the generation of a wide variety of UWB pulses, including polarity-inverted monocycles and doublets. A key advantage in exploiting XPM was the chirp erasure of the input optical pulses, because XPM depends solely on the pulses intensity. Thus, shaped UWB optical pulses have a good tolerance to dispersion over fiber and are more suitable for long-distance transmission in UWB over fiber communication systems.

Very recently, Chen et al [37] have reported a step towards the integration of a reconfigurable MWP filter concept in [14], by exploiting an FWM process in a silicon nanowire, instead of using HLNF. The silicon waveguide $(h=220 \mathrm{~nm}, w=650 \mathrm{~nm}$, length $=12 \mathrm{~mm}$ ) was dispersion engineered to yield broadband FWM. They generated 2 idlers with measured conversion efficiency of $-25 \mathrm{~dB}$. These wavelengths taps were then modulated and amplitude controlled using a waveshaper. The used $4 \mathrm{~km}$ of single mode fiber (SMF) as the dispersive medium required to generate time delay between the taps. The measured the response of a 4-tap MWP filter and demonstrated that the $12 \mathrm{~mm}$ silicon nanowire can be used to replace $1 \mathrm{~km}$ of silica HNLF without compromising the quality of the MPF response.

\section{OUTLOOK}

We believe that the growth of nonlinear IMWP will strongly depend on two factors: the efficiency of the associated on-chip nonlinear processes, and the integrability of the nonlinear platform of choice. Most of the previously reported results were demonstrated in a highly nonlinear chalcogenide glass. This platform enabled highly efficient nonlinear processes but it is relatively limited in terms integration potential. CMOS compatible platforms such as silicon or silicon nitride are more attractive due to the moderate to high nonlinearity but more importantly their potential of integrating linear and nonlinear optics components as well as modulators and detectors either monolithically, or through hybrid integration schemes. This also applies, for example, to the high performance notch filter concept reported in [33-35]. It is very attractive to implement such as scheme with on-chip SBS in silicon/silicon nitride hybrid optical structure as shown in [38].

In the view of generating the multipurpose ASP, we believe incorporating photonic crystal structures [39] will be highly attractive for a number of reasons. This structure can be made highly dispersive, to replace the optical fiber spool for the time delay element required in most signal processing tasks.

\section{CONCLUSIONS}

In this paper we introduced an exciting new concept in microwave photonics, where on-chip linear and non-linear processes are combined to enable unprecedented RF signal processing capabilities. We also offered perspective on key factors that will determine the growth of this field. 


\section{ACKNOWLEDGMENT}

D.M. would like to thank Prof. Jose Capmany and Maurizio Burla for useful discussions. The authors thank Blair Morrison, Ravi Pant, Duk-Yong Choi, Steve Madden, Barry LutherDavies, Kang Tan, and Chris Roeloffzen for their contribution.

\section{REFERENCES}

[1] D. Marpaung, C. Roeloffzen, R. Heideman, A. Leinse, S. Sales, and J. Capmany, "Integrated microwave photonics", Laser \& Photon. Rev. 7, 506, 2013.

[2] G. P. Agrawal, Nonlinear Fiber Optics, Academic, San Diego, Calif, USA, 3rd edition, 2002.

[3] Y. Okawachi, M. S. Bigelow, J. E. Sharping, Z. M. Zhu, A. Schweinsberg, D. J. Gauthier, R. W. Boyd, and A. L. Gaeta, "Tunable all-optical delays via Brillouin slow light in an optical fiber," Phys. Rev. Lett. 94, 153902, 2005.

[4] B.J. Eggleton, T.D. Vo, R. Pant, J. Schroeder, M.D. Pelusi, D. Yong Choi,S.J. Madden, and B. Luther-Davies, "Photonic chip based ultrafast optical processing based on high nonlinearity dispersion engineered chalcogenide waveguides", Laser \& Photon. Rev., 6: 97-114, 2012.

[5] A.E. Willner, O.F. Yilmaz, Jian Wang; Xiaoxia Wu A. Bogoni, Lin Zhang, S.R. Nuccio, "Optically Efficient Nonlinear Signal Processing," IEEE Journal of Selected Topics in Quantum Electronics, vol.17, no.2, pp.320,332, 2011.

[6] C. Caloz, S. Gupta, Q. Zhang, and B. Nikfal, "Analog Signal Processing", arXiv:1307.2618 [physics.optics], 2013.

[7] T.K. Woodward, A. Agarwal, T. Banwell, P. Toliver, B.J. Luff, D. Feng, P. Dong, D.C. Lee, N-N. Feng, and M. Asghari, "Systems perspectives on optically-assisted RF signal processing using silicon photonics," Microwave Photonics, IEEE MWP 2011., pp.377,380, 18-21 Oct. 2011.

[8] M. Burla, D. A. I. Marpaung, L. Zhuang, C. G. H. Roeloffzen, M. R. Khan, A. Leinse, M. Hoekman, and R. G. Heideman, "On-chip CMOS compatible reconfigurable optical delay line with separate carrier tuning for microwave photonic signal processing," Opt. Express 19(22), 21475 21484, 2011.

[9] D. Marpaung, C. Roeloffzen, A. Leinse, and M. Hoekman, “A photonic chip based frequency discriminator for a high performance microwave photonic link," Opt. Express18(26), 27359-27370, 2010.

[10] M. H. Khan, H. Shen, Y. Xuan, L. Zhao, S. Xiao, D. E. Leaird, A. M. Wiener, and M. Qi, "Ultrabroad-bandwidth arbitrary radiofrequency waveform generation with a silicon photonic chip-based spectral shaper," Nat. Photonics 4(2), 117-122, 2010

[11] I. Gasulla and J. Capmany,"Analog Filtered links: A unifying approach for Microwave Photonic systems," ICTON 2012, 2-5 July 2012.

[12] C-S. Brès, S Zlatanovic, A.O.J. Wiberg, and S. Radic, "Reconfigurable parametric channelized receiver for instantaneous spectral analysis," Opt. Express 19, 3531-3541, 2011.

[13] C-S. Bres, S. Zlatanovic, A.O.J. Wiberg, J.R. Adleman, C.K. Huynh, E.W. Jacobs, J.M. Kvavle, and S.Radic,"Parametric Photonic Channelized RF Receiver," IEEE Photonics Technology Letters, vol.23, no.6, pp.344,346, 2011.

[14] B. Vidal, J. Palaci, and J. Capmany, "Reconfigurable Photonic Microwave Filter Based on Four-Wave Mixing," IEEE Photonics Journal, , vol.4, no.3, pp.759,764, 2012.

[15] S. Khaleghi, M.R. Chitgarha, O.F. Yilmaz, M. Tur, M. W. Haney, C. Langrock, M. M. Fejer, and A. E. Willner, "Experimental performance of a fully tunable complex-coefficient optical FIR filter using wavelength conversion and chromatic dispersion," Opt. Lett. 37, 34203422,2012

[16] L. Zhuang, M. Hoekman, W.P. Beeker, A. Leinse, R. Heideman, P. van Dijk, and C. Roeloffzen, Novel low-loss waveguide delay lines using Vernier ring resonators for on-chip multi- $\lambda$ microwave photonic signal processors. Laser \& Photon. Rev, 2013.

[17] L, Zhuang, D. A. I. Marpaung, M. Burla, W. P. Beeker, A. Leinse, and C. G. H. Roeloffzen, "Low-loss, high-index-contrast Si3N4/SiO2 optical waveguides for optical delay lines in microwave photonics signal processing," Opt. Express 19(23), 23162-23170, 2011.

[18] Ikeda, K., Saperstein, R. E., Alic, N. \& Fainman, Y. Thermal and Kerr nonlinear properties of plasma-deposited silicon nitride/silicon dioxide waveguides. Opt. Express 16, 12987-12994, 2008.

[19] D. J. Moss, R. Morandotti, A. L. Gaeta, and M. Lipson, "New CMOS compatible platforms based on silicon nitride and Hydex for nonlinear optics," Nat. Photonics 7, 597-607, 2013.

[20] J. S. Levy, A. Gondarenko, M. A. Foster, A. C. Turner-Foster, A. L. Gaeta, and M. Lipson, "CMOS-compatible multiple-wavelength oscillator for on-chip optical interconnects," Nat. Photonics 4(1), 37-40 2010

[21] J. S. Levy, A. Gondarenko, A. C. Turner-Foster, M. A. Foster, A. L. Gaeta, and M. Lipson, "Four-wave mixing in integrated silicon nitride waveguides," presented at the CLEO 2009, Baltimore, MD, CMFF5

[22] A. Biberman, B. G. Lee, A. C. Turner-Foster, M. A. Foster, M Lipson, A. L. Gaeta, and K. Bergman, "Wavelength multicasting in silicon photonic nanowires," Opt. Express 18, 18047-18055, 2010.

[23] M. Pu, H. Hu, H. Ji, M. Galili, L. K. Oxenløwe, P. Jeppesen, J. M. Hvam, and K. Yvind, "One-to-six WDM multicasting of DPSK signals based on dual-pump four-wave mixing in a silicon waveguide," Opt. Express 19, 24448-24453, 2011.

[24] W. Bogaerts, P. De Heyn, T. Van Vaerenbergh, K. DeVos, S. K. Selvaraja, T. Claes, P. Dumon, P. Bienstman, D. Van Thourhout, and R. Baets, "Silicon microring resonators," Laser Photonics Rev.6(1), 47-73, 2012

[25] X. S. Yao, "Brillouin selective sideband amplification of microwave photonic signals," IEEE Photon. Technol. Lett., vol. 10, no. 1, pp. 138140, Jan. 1998.

[26] A. Loayssa, D. Benito, and M. J. Garde, "Optical carrier Brillouin processing of microwave photonic signals," Opt. Lett. 25, 1234-1236, 2000

[27] R. Pant, C. G. Poulton, D-Y Choi, H. Mcfarlane, S. Hile, E. Li, L. Thevenaz, B. Luther-Davies, S. J. Madden, and B. J. Eggleton, "On-chip stimulated Brillouin scattering," Opt. Express 19, 8285-8290, 2011

[28] R. Pant, A. Byrnes, C.G. Poulton, E. Li, D.Y. Choi, S. Madden, B. Luther-Davies, and B. J. Eggleton., "Photonic-chip-based tunable slow and fast light via stimulated Brillouin scattering," Opt. Lett. Vol 37, pp. 969-971, 2012.

[29] A. Byrnes, R. Pant, E. Li, D.Y. Choi, C.G. Poulton, S. Fan, S. Madden, B. Luther-Davies, and B. J. Eggleton, "Photonic chip based tunable and reconfigurable narrowband microwave photonic filter using stimulated Brillouin scattering," Opt. Express 20, 18836-18845 2012.

[30] D. Marpaung, R. Pant, B. Morrison, E. Li, D.Y. Choi, S. Madden, B. Luther-Davies, and B.J. Eggleton, "Microwave photonic notch filter using on-chip stimulated Brillouin scattering", Proc. CLEO-PR 2013 , paper ThB2-6, Kyoto, Japan, 30 June-4 July 2013.

[31] B. Morrison, D. Marpaung, R. Pant, E. Li, D-Y. Choi, S. Madden, B Luther-Davies, B. J. Eggleton, Tunable microwave photonic notch filter using on-chip stimulated Brillouin scattering, Opt. Comm., http://dx.doi.org/10.1016/j.optcom.2013.09.065i (2013).

[32] D.R. Jachowski., "Compact, frequency-agile, absorptive bandstop filters," IEEE MTT-S IMS 2005, 12-17 June 2005

[33] D. Marpaung, R. Pant, B. Morrison, and B.J. Eggleton, "Frequency agile microwave photonic notch filter with anomalously-high stopband rejection", Opt. Lett. 38, 4300-4303 (2013).

[34] D. Marpaung, B. Morrison, R. Pant, D. Y. Choi, S. Madden, B. LutherDavies, and B. J. Eggleton, "A Tunable RF Photonic Notch Filter with Record 55 dB Suppression using Sub-1 dB On-Chip Brillouin Gain," in Frontiers in Optics 2013, Postdeadline paper FW6B.9 (2013)

[35] D. Marpaung, R. Pant, B. Morrison, Chris Roeloffzen, Arne Leinse, Marcel Hoekman, Rene Heideman, and B.J. Eggleton, " $\mathrm{Si}_{3} \mathrm{~N}_{4}$ ring resonator-based microwave photonic notch filter with an ultrahigh peak rejection", Opt. Express 21, 23286-23294 (2013).

[36] K. Tan, D. Marpaung, R. Pant, F. Gao, E.Li, J. Wang, D-Y. Choi, S. Madden, B. Luther-Davies, J. Sun, and B. J. Eggleton, "Photonic-chipbased all-optical ultra-wideband pulse generation via XPM and 
birefringence in a chalcogenide waveguide," Opt. Express 21, 20032011, 2013.

[37] L. R. Chen, J. Li, M. Spasojevic, and R. Adams, "Nanowires and sidewall Bragg gratings in silicon as enabling technologies for microwave photonic filters," Opt. Express 21, 19624-19633 (2013).
[38] H. Shin, Z. Wang, W. Qiu, R. Jarecki, J. Cox, T. Olsson, and P. T. Rakich, "Tailorable stimulated Brillouin scattering at nanoscale silicon waveguides", Nat. Comm. 4, 1944, (2013)

[39] J. Sancho, J. Bourderionnet, J. Lloret, S. Combrié, I. Gasulla, S. Xavier, S. Sales, P. Colman, G. Lehoucq, D. Dolfi, J. Capmany and A. De Rossi, "Integrable microwave filter based on a photonic crystal delay line". Nat. Comm. 3, 1075, 2012. 